\title{
APLICAÇÃO DO MÉTODO FUZZY NA CLASSIFICAÇÃO DA ZONA DE CONVERGÊNCIA DO ATLÂNTICO SUL NO SUL DA AMAZÔNIA
}

\author{
SAMUEL OLIVEIRA VIEIRA ${ }^{1}$, NILOMAR VIEIRA OLIVEIRA ${ }^{1}$, PRAKKI SATYAMURTY ${ }^{2,3}$, RITA \\ VALERIA ANDREOLI ${ }^{2}$
}

\author{
${ }^{1}$ Universidade Federal do Amazonas, Instituto de Educação, Agricultura e Ambiente e Instituto de Ciências \\ Exatas, Manaus, AM, Brasil \\ ${ }^{2}$ Universidade do Estado do Amazonas, Escola Superior de Tecnologia, Manaus, AM, Brasil \\ ${ }^{3}$ Instituto Nacional de Pesquisas Espaciais, São José dos Campos, SP, Brasil
}

samhuel.matematico@gmail.com,nilomar@gmail.com,saty.prakki@gmail.com,rasouza@uea.edu.br

Recebido Maio de 2013 - Aceito Dezembro de 2013

\begin{abstract}
RESUMO
A Zona de Convergência do Atlântico Sul (ZCAS) influencia a variabilidade da precipitação no sul da bacia Amazônica. Nesse sentido, foi aplicado o Método da Lógica Fuzzy de modo a ter um mecanismo de alerta para as chuvas acima da média climatológica devido à ZCAS (classificada como oceânica, costeira ou Amazônica) sobre o Sul da Amazônia, nos meses de novembro a março no período 1999 a 2010. Um algoritmo objetivo na identificação da ocorrência de ZCAS foi esquematizado e a classificação em termos linguísticos permitiu categorizar em diferentes tipos. Durante os períodos de ZCAS, a possibilidade de ocorrer chuvas fortes diárias ( $>20 \mathrm{~mm}$ ) é mais de duas vezes maior do que a climatologia. ZCAS Amazônicas e costeiras produzem aproximadamente 71,4\% da precipitação, referentes aos meses de janeiro a março representando 64\% dos episódios atuantes no Sul da Amazônia. Palavras-chave: Lógica Fuzzy, classificação de ZCAS, precipitação.
\end{abstract}

\begin{abstract}
APPLICATION OF FUZZY METHOD IN THE CLASSIFICATION OF THE SOUTH ATLANTIC CONVERGENCE ZONE IN THE AMAZON REGION

The South Atlantic Convergence Zone (SACZ) influences the variability of precipitation in South Amazon Basin. In this sense, we applied the Fuzzy Logical Method in order to have a mechanism of warning for the precipitation above the climatological average due to the SACZ (classified as oceanic, coastal or Amazon) over the Southern Amazon region, during the months from November to March in the period from 1999 to 2010 . An objective algorithm to identify the occurrence of SACZ is designed and the classification in linguistic terms allows categorizing the SACZ in different types. During the periods of SACZ, the possibility of daily heavy rains $(>20 \mathrm{~mm})$ is twice greater than the climatology. Amazonian and coastal SACZ produce nearly $71.4 \%$ of the precipitation during the months from January to March, representing 64\% of the active events in Southern Amazon.
\end{abstract}

Keywords: Fuzzy Logical Method, SACZ classification, precipitation.

\section{INTRODUÇÃO}

Um dos sistemas meteorológicos de escala regional que influência o tempo sobre grande parte da América do Sul (AS) tropical é a Zona de Convergência do Atlântico Sul (ZCAS). Esse sistema desempenha um papel importante no Sistema de monção da América do Sul (Vera et al., 2006). Na AS, as estações chuvosa e seca são bem definidas e o transporte de umidade do Oceano Atlântico para o continente tem uma forte variabilidade sazonal (Satyamurty et al., 2012).
A banda de nebulosidade associada à ZCAS se estende da Bacia Amazônica ao Oceano Atlântico Sul subtropical (Kodama, 1992; Satyamurty et al., 1998) e afeta muitas regiões do Brasil com chuvas fortes e intensas, com duração entre 4 e 15 dias. Apesar de sua extensão espacial, nem todos os eventos de ZCAS afetam a Bacia Amazônica (De Oliveira Vieira et al., 2012), exceto àqueles especialmente fortes e intensos sobre o interior do continente Sul Americano (Carvalho et al., 2004), que influenciam fortemente o clima dessa região. 
A estação chuvosa na Amazônia se estende de novembro a abril, com uma média de $2200 \mathrm{~mm}$ de precipitação anual sobre a Amazônia; cerca de 70\% de chuvas ocorre nesse período (Rao e Hada, 1990; Rao et al., 1996). Muitos estudos (Figueroa et al., 1995; Satyamurty et al., 1998) têm mencionado que os episódios de ZCAS fortes ocorrem de três a cinco vezes por ano durante o verão austral.

A busca constante de vários pesquisadores em entender mecanismos de previsão de chuvas sobre uma determinada região, em particular as fortes e intensas a exemplo das observadas em eventos de ZCAS, incentivou a utilização neste trabalho da metodologia Fuzzy. A Lógica Fuzzy é uma técnica muito útil na solução de problema com extensa aplicabilidade. É usada atualmente nas áreas de negócios, sistemas de controle, eletrônica e engenharia de tráfego, meteorologia, entre outras aplicações. A técnica pode ser usada para gerar soluções para os problemas com base em "vago, ambíguo, qualitativa, informação incompleta ou imprecisa". Há muitos problemas meteorológicos de previsão que se encaixam na descrição anterior, onde rigorosas soluções analíticas não existem, ou seja, entre uma categoria seca e chuvosa existem subcategorias que devem ser levadas em conta, e que estão fora desses extremos. Lógica Fuzzy é uma extensão da teoria dos Conjuntos Fuzzy, que foi desenvolvido há aproximadamente 45 anos atrás.

A aplicação dessa teoria em estudos meteorológicos vem crescendo nos últimos anos por todo o mundo. Dentre eles, Shao (2000), mapeou características térmicas da atmosfera, Hicks et al. (2004) apresentou um sistema com base em Lógica Fuzzy para automatizar previsões de tempo de curto prazo para fins aeronáuticos e Da Silva et al. (2007) utilizaram a teoria de conjuntos difusos (método Fuzzy) com o objetivo de unificar as informações climáticas de consenso subjetivo, visando a obtenção de uma previsão determinística de um Índice Regional de Chuvas da Quadra Chuvosa (IRCQC) da Região Semi-Árida do Nordeste do Brasil.

Neste trabalho propõe-se aplicar o método Fuzzy para estudar os tipos de ZCAS que influenciam a precipitação no Sul da Amazônia, visando classificar esses eventos em relação a sua intensidade (fraco, forte e intenso) utilizando como dados de entrada precipitações climatológicas e àquelas associadas aos eventos de ZCAS.

\section{DADOS E METODOLOGIA}

\section{1. Dados}

Os dados de precipitação utilizados para este estudo correspondem aos 55 meses de novembro a março dos anos de 1999 até 2010. A área de estudo (área alvo) está compreendida entre as latitudes de $14^{\circ} \mathrm{S}$ a $6^{\circ} \mathrm{S}$ e longitudes $66^{\circ} \mathrm{W}$ a $50^{\circ} \mathrm{W}$ e pode ser observada na Figura 4.

Os dados de precipitação global são disponibilizados no sítio http://disc2.nascom.nasa.gov/Giovanni/tovas/, e se referem às precipitações diárias oriundas do satélite Tropical Rainfall Measuring Mission (TRMM) com resolução espacial de $0,25^{\circ}$ x $0,25^{\circ}$. Além disso, precipitações observadas (estações pluviométricas), em Canarana, Gleba Celeste, Matupá, Lábrea, Manicoré e São José do Rio Claro foram obtidas do Banco de Dados Meteorológicos para Ensino e Pesquisa (BDMEP) do Instituto Nacional Meteorologia (INMET).

Os dados estimados de Radiação de Onda Longa (ROL) disponibilizados pela "National Oceanic and Atmospheric Administration" (NOAA), utilizados para o esse estudo possuem resolução espacial de $2,5^{\circ}$ por $2,5^{\circ}$ de latitude por longitude, e estão disponíveis no sitio http://www.esrl.noaa.gov/psd/data/ gridded/data.interp_OLR.html. Os dados servem como "proxy" para caracterizar a atividade convectiva associada aos eventos de ZCAS.

\subsection{Metodologia}

\section{2. 1 Coeficiente de eficiência dos episódios de ZCAS}

Inicialmente, para criar os conjuntos Fuzzy e aplicar o método à variável precipitação sobre o sul da Amazônia, a fim de classificar os tipos de ZCAS que influenciam as chuvas acima da média climatológica, definiu-se um Coeficiente de eficiência $(\alpha)$ que traduz a intensidade de chuva diárias dos episódios de ZCAS que afetam o Sul da Amazônia. Para isso, utilizou-se a série de dados observados descrita por De Oliveira Vieira et al., (2012) para os meses NDJFM durante o período de 1999 a 2010.

$\mathrm{O}$ Coeficiente de Eficiência diz respeito à frequência diária das chuvas intensas quando os eventos de ZCAS estão atuando no sul da Amazônia e é definido como segue:

$$
\alpha=(\mathrm{m} / \mathrm{M}) \mathrm{x}(\mathrm{D} / \mathrm{d})
$$

em que $\mathrm{m}$ é número total de casos de chuvas intensas (maior ou igual ao limiar) durante o período de atuação de ZCAS, M corresponde ao número de todos os dias de chuvas fortes nos 55 meses, D é o número de dias do período de 5 meses de NDJFM desses 11 anos de estudo, d é o número total de dias da atuação de ZCAS. Se $\alpha>1$ significa que o evento de ZCAS contribui para propiciar chuvas intensas com maior frequência em relação à climatológica.

Se a proporção de $\mathrm{m}$ em relação a $\mathrm{d}$ for igual à proporção de $\mathrm{M}$ em relação a $\mathrm{D}$, ou seja, $\mathrm{m} / \mathrm{M}=\mathrm{d} / \mathrm{D}$, então $\alpha$ $=1$, significando que o número de dias com chuvas intensas durante os episódios de ZCAS não é proporcionalmente maior em relação a climatologia. Agora, se $\alpha>1$, significa que os 
episódios de ZCAS apresentam proporcionalmente maior número de chuvas intensas do que a média de verão.

Considerando o limiar de $20 \mathrm{~mm} / \mathrm{dia}$ (escolhido de forma subjetiva com base na experiência diária), os dias de ZCAS em que houve precipitação maior ou igual a esse limiar corresponde a $\mathrm{m}=63$ dias e $\mathrm{d}=232$, sendo $\mathrm{d}$ o número total de dias com atuação dos 28 episódios no período de estudo. $\mathrm{M}=195$ corresponde ao total de dias com precipitações $\geq 20$ $\mathrm{mm} /$ dia e o número $\mathrm{D}=1661$, o total de dias em 55 meses de NDJFM no período de 1999 a 2010.

Assim, depois de efetuado o cálculo com os dados observados, obteve-se o valor do Coeficiente de Eficiência $\alpha=$ 2,31, o que significa que a contribuição dos eventos de ZCAS em produzir chuvas fortes ou intensas é proporcionalmente superior ao dobro de dias da climatologia.

Em seguida, é necessário conhecer quais os tipos de ZCAS em relação a sua área de atuação, ou seja, ZCAS oceânica, costeira e amazônica (ZCO, ZCC e ZCAM, respectivamente) que influenciam o sul da Amazônia com chuva forte ou intensa acima da climatologia. Para isso utiliza-se o método Fuzzy.

\subsubsection{Modelagem Fuzzy na classificação de ZCAS}

\subsubsection{Sistema Fuzzy}

Seres humanos são capazes de lidar com processos bastante complexos baseados em informações imprecisas ou aproximadas. A Teoria dos Conjuntos Fuzzy e os Conceitos de Lógica Fuzzy, concebida por Zadeh (1965 e 1978), podem ser utilizados para traduzir em termos matemáticos a informação imprecisa expressa por um conjunto de termos de regras linguísticas (Da Silva et al., 2007).
Esta subjetividade nos permite classificar as ZCAS em ZCO, ZCC e ZCAM, através de uma expressão matemática que indica o grau com que o elemento $\mathrm{x}$ de um conjunto $\mathrm{U}$ está em concordância com o conceito que caracteriza os elementos $\mathrm{x}$ no subconjunto A contido em U. Um subconjunto fuzzy A de um conjunto $U$ é caracterizado por uma função de pertinência $\mu \mathrm{A}: \mathrm{U} \rightarrow[0,1]$, onde $\mu \mathrm{A}(\mathrm{x})$ mede a probabilidade do elemento $\mathrm{x}$ pertencer ao subconjunto $\mathrm{A}$, ou seja, o valor de $\mu \mathrm{A}(\mathrm{x}) \in$ $[0,1]$. A pertinência ou não pertinência de um elemento em A é indicado por $\mu \mathrm{A}(\mathrm{x})=1 \mathrm{e} \mu \mathrm{A}(\mathrm{x})=0$, respectivamente (Sugeno, 1988).

\section{2. 2. 2 Sistema de Mamdani}

Aqui, apresenta-se um resumo do método Mamdani de inferência segundo Barros (1997). Espera-se que a cada entrada crisp (um número real ou n-upla de números reais) faça corresponder uma saída crisp, e em geral, um sistema Fuzzy faça corresponder a cada entrada uma saída. Neste caso, um sistema Fuzzy é uma função de Rn em R, construída por uma metodologia específica de acordo com 3 módulos (Figura 1).

1. Módulo de fuzzificação: modela matematicamente a informação das variáveis de entrada por meio de conjuntos fuzzy. É neste módulo que se mostra a grande importância do especialista no processo a ser analisado, pois a cada variável de entrada devem ser atribuídos termos linguísticos que representam os estados desta variável e, a cada termo linguístico, deve ser associado a um conjunto fuzzy por uma função de pertinência. É nesse módulo que se armazenam as variáveis e suas classificações linguísticas;

2. Módulo de inferência: é onde se definem os conectivos lógicos usados para estabelecer a relação fuzzy que modela a

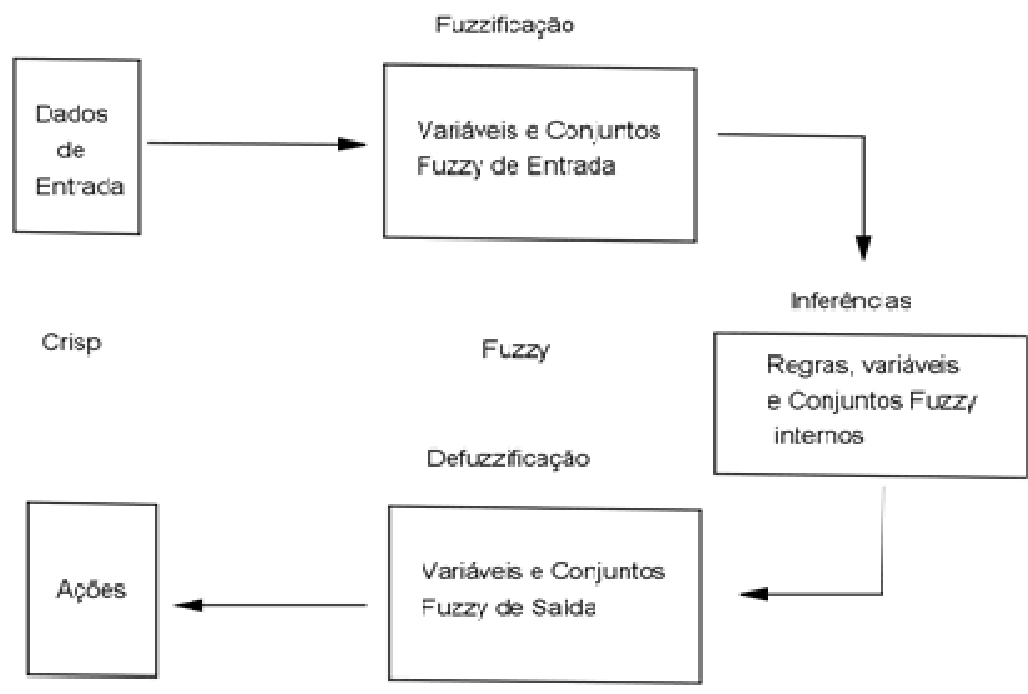

Figura 1 - Estrutura de um controlador de lógica fuzzy. 
base de regras. É deste módulo que depende o sucesso do sistema Fuzzy já que ele fornecerá a saída (controle) fuzzy a ser adotado pelo controlador a partir de cada entrada fuzzy;

3. Módulo de defuzzificação: que traduz o estado da variável de saída fuzzy para um valor numérico.

\section{2. 2. 3 Número Fuzzy}

Na literatura aparecem vários números Fuzzy, os mais comuns são triangulares, trapezoidais e em forma de sino. Dentre eles, o que melhor se ajustou ao nosso modelo foi a trapezoidal, uma vez que trabalha-se com as médias centralizadas em um dado intervalo, cujos valores extremos estão relacionados com as funções médias e desvios padrão. Assim, defini-se como um número Fuzzy Trapezoidal a função de pertinência $\mu \mathrm{A}(\mathrm{x})$ da forma:

1. $(x-a) /(b-a)$, se $a<=x<b$;

2. 1 , se $\mathrm{b}<=\mathrm{x}<=\mathrm{c}$;

3. $(\mathrm{d}-\mathrm{x}) /(\mathrm{d}-\mathrm{c})$, se $\mathrm{c}<\mathrm{x}<=\mathrm{d}$;

4. 0 , caso contrário, onde $\mu \mathrm{A}(\mathrm{x})$ está definido no intervalo fechado $\mathrm{v}=[\mathrm{a}, \mathrm{d}]$.

A Figura 2 destaca o número trapezoidal pela função de pertinência definida em um intervalo $\mathrm{v}=[\mathrm{a}, \mathrm{b}) \cup[\mathrm{b}, \mathrm{c}) \cup[\mathrm{c}, \mathrm{d}]$, onde cada intervalo corresponde aos valores de chuvas normais (CN) ou de ZCAS (CZ) relacionadas a cada categoria, por exemplo, nos intervalos semi-fechados $[\mathrm{a}, \mathrm{b})$ as chuvas de $\mathrm{CN}$ são consideradas fracas, $[\mathrm{b}, \mathrm{c})$ são médias e $[\mathrm{c}, \mathrm{d}]$ são fortes. Assim, se $\mu \mathrm{A}(\mathrm{x})=(\mathrm{x}-\mathrm{a}) /(\mathrm{b}-\mathrm{a})$, então o valor de $\mathrm{x}$ pertence ao domínio $[\mathrm{a}, \mathrm{b})$ cuja a imagem pertence à parte esquerda do trapézio, ou seja, $\mu \mathrm{A}$ (a) $\leq \mu \mathrm{A}(\mathrm{x})<\mu \mathrm{A}$ (b) onde $\mu \mathrm{A}$ (a) e $\mu \mathrm{A}$ (b) são os valores limitantes nas bases inferior e superiores do lado esquerdo do trapézio. De modo semelhante $\mu \mathrm{A}$ (b) e $\mu \mathrm{A}$ (c) representam os valores que limitam a base superior do trapézio, e finalmente os valores de $\mu \mathrm{A}$ (c) e $\mu \mathrm{A}$ (d) representam as bases superior e inferior do lado direito do trapézio.
Os operadores máximo e mínimo são definidos abaixo, respectivamente:

1. $(\mu \mathrm{A} \cup \mu \mathrm{B})(\mathrm{x})=\max (\mu \mathrm{A}(\mathrm{x}), \mu \mathrm{B}(\mathrm{x})), \forall \mathrm{x} \in \mathrm{U}$;

2. $(\mu \mathrm{A} \cap \mu \mathrm{B})(\mathrm{x})=\min (\mu \mathrm{A}(\mathrm{x}), \mu \mathrm{B}(\mathrm{x})), \forall \mathrm{x} \in \mathrm{U}$.

A escolha dos operadores máximo e mínimo na definição da união e intersecção fuzzy se justifica, pelo fato de que quase todas as operações clássicas dos conjuntos são preservadas. Os valores de entrada são definidos através dos domínios e termos linguísticos atribuídos as intensidades da precipitação para todo o período ou para apenas um mês de analise. Sendo assim, foram calculados a precipitação média climatológica sobre a área de estudo (PmcTA) e desvio padrão ( $\sigma \mathrm{TA})$, precipitação média dos 28 episódios de ZCAS sobre a área de estudo (PmZCTA) e desvio padrão desse episódios $(\sigma Z C T A)$, onde TA representa a precipitação média sobre a área de estudo, calculada com base na série temporal obtida pela metodologia descrita por De Oliveira Vieira et al. (2012).

Os termos linguísticos fraca, média e forte sobre a chuva climatológica ou normal $(\mathrm{CN})$ foram definidos da seguinte maneira:

a) Se $\mathrm{CN}$ estiver entre $0<\mathrm{CN}<=$ PmcTA - $(\sigma / 2) \mathrm{TA}$ é considerada chuva fraca;

b) $\mathrm{Se} \mathrm{CN}$ estiver entre PmcTA - $(\sigma / 2) \mathrm{TA}<\mathrm{CN}<=$ PmcTA $+(\sigma / 2)$ TA é chamada de chuva média;

c) Se CN estiver entre PmcTA $+(\sigma / 2) \mathrm{TA}<\mathrm{CN}$ é considerada chuva forte. Caso contrário, está se falando de intervalos de transição, ou seja, o valor $\mu$ pertence a duas categorias, aumentando ou diminuindo o grau de probabilidade de uma categoria.

Os conjuntos dos termos linguísticos para os 28 episódios de ZCAS utilizando a PmZCTA e o $\sigma Z C T A$ foram obtidos de modo análogo de acordo com os termos linguísticos (fraca, forte e intensa) de ZCAS. A classificação dos eventos de ZCAS em oceânica, costeira e Amazônica obedece aos mesmos critérios, mas o que determina os intervalos (categorias) são as porcentagens segundo a média e desvio padrão.

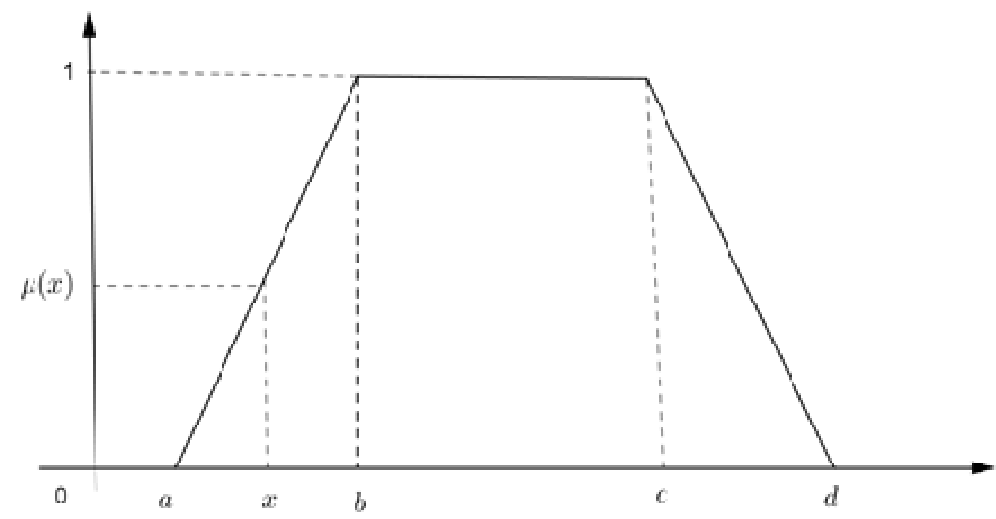

Figura 2 - Representação do número trapezoidal $\mu(\mathrm{x})$ em suas respectivas base ou intervalos. Eixo y mede o grau de pertinência e o eixo x os intervalos das precipitações. 


\section{2. 2. 4 Dados de entrada}

Os dados de entrada e saída representam de forma esquemática o sistema Fuzzy, através dos dados de precipitações normais ( $\mathrm{CN} \mathrm{mm/dia)} \mathrm{e} \mathrm{dos} 28$ episódios de ZCAS referentes ao período de estudo. As relações entre os dados de entrada e saídas dependem da Base de regras e Inferências.

1. Entrada1: $\mathrm{CN}$ - é a chuva para todo o período de estudo na área alvo, com a média climatológica igual a $9,4 \mathrm{~mm} / \mathrm{dia} \mathrm{e}$ o desvio igual à metade do desvio padrão $\sigma / 2=2,2 \mathrm{~mm} /$ dia.

(a) Termos Linguísticos: fraca, média e forte;

i. fraca $=\left[\begin{array}{llll}0 & 0 & 5 & 7,2\end{array}\right]$

ii. média $=[57,211,613,8]$

iii. forte $=[11,613,826,626,6]$

Universo (CN): 0 a $26,6 \mathrm{~mm} /$ dia.

Os dois valores 0 no intervalo de chuvas fracas (i) correspondem às bases inferior e superior do trapézio do lado esquerdo e os valores 5 e 7,2 correspondem às bases superior e inferior do lado direito do mesmo. Esses valores 5 e 7,2 em (i) representam a média climatológica menos o desvio padrão e assim como a média climatológica menos a metade do desvio padrão. De modo análogo, os valores dos intervalos de Entrada2 e Saída foram manipulados.

2. Entrada2: CZ - é a chuva de todos os dias dos eventos de ZCAS.

(a) Termos Linguísticos: fraca, forte e intensa;

i. fraca [0 009,2 11,4]

ii. forte $[9,211,415,818]$

iii. Intensa [15,8 18 26,6 26,6]

Universo (CZ): 0 a $26,6 \mathrm{~mm} /$ dia.

3. Saída: ZCAS oceânica e/ou costeira e/ou Amazônica corresponde aos valores das porcentagens dos eventos. Nesses intervalos utiliza-se o valor da percentagem de atuação de ZCAS na área alvo, para definir os intervalos em (i), (ii) e (iii).

(a) Termos Linguísticos: oceânica, costeira e Amazônica;

i. oceânica $[007,14$ 17,14]

ii. costeira [7,14 17,14 40,14 50,14]

iii. Amazônica [40,14 50,14 100 100]

Universo(ZCAS): $[0, \ldots, 100] \%$.

A condicional "se" chuvas normais $(\mathrm{CN})$ é fraca "e" chuvas de ZCAS (CZ) também é fraca, "então" a ZCAS atuante no Sul da Amazônia é oceânica, essa condicional possui uma função de pertinência $\mu$ (fraca, fraca) : $\mathrm{CN}$ x CZ $\rightarrow[0,1]$, onde $\mu$ mede o grau de verdade da relação de implicação entre os conjuntos $\mathrm{CN}$ (fraca) e $\mathrm{CZ}$ (fraca) que corresponde ao tipo de ZCAS, o símbolo " $\wedge$ " representa a operação interseção dos conjuntos.

A Base de Regras e o Método de Inferência estão relacionados com a probabilidade da função de pertinência pertencer a cada intervalo (conjunto) determinado pelas precipitações médias diárias e desvios padrões de $\mathrm{CN}$ e $\mathrm{CZ}$ sobre o sul da Amazônia, definidos anteriormente. CN também pode ser analisada pela chuva do mês em que ocorreu o evento de ZCAS. Assim, a base de Regra no implemento do algoritmo é definido como segue:

Base de Regra

1. Se (CN é Fraca)e (CZé Fraca) então (ZCAS é oceânica); 2. Se (CNé Fraca) e (CZ é Forte) então (ZCAS é oceânica); 3. Se(CNé Fraca)e (CZéIntensa) então (ZCAS é costeira); 4. Se(CNé Média)e (CZéFraca) então (ZCAS é oceânica); 5. Se (CN é Média)e (CZ é Forte) então (ZCAS é costeira); 6. Se (CN é Média) e (CZ é Intensa) então (ZCAS é continental);

7. Se (CN é Forte) e (CZ é Fraca) então (ZCAS é costeira); 8. Se(CNéForte)e(CZéForte)então(ZCASécontinental); 9. Se(CNéForte)e(CZéIntensa)então(ZCASécontinental).

Nessa etapa, utilizou-se o Método do centro de gravidade. Maiores detalhes ver Mamdani e Assillan (1975) e Da Silva et al. (2007). Portanto, a operação "e" lógica das variáveis linguísticas será resolvida através do operador mínimo. Por exemplo, se CN é fraca e CZ é fraca, então será resolvida através do operador mínimo entre fraca e fraca.

$\mathrm{Na}$ prática, as operações para se obter o algoritmo de controle consistiram nos seguintes procedimentos: 1. Para cada fator da parte "Se" da regra obtêm-se um grau de participação do valor de entrada para cada função de associação ou termo linguístico; 2. O mínimo valor dos graus de participação obtidos em 1 é o grau de participação da parte "Se" (antecedente); 3. Aplicar um limitador na função de associação da parte "então" através do fator obtido; 4. Fixando CN para todas as condições e para cada uma delas pode variar $\mathrm{CZ}$ nas 3 condições de cada etapa nas regras e obter a soma lógica "e" das funções de pertinência associação, dadas pelas partes "então" (consequente) de cada regra. 5. Calcular a defuzzificação da função de pertinência resultante e obter o valor de saída.

\section{RESULTADOS}

\section{1 Análises do método Fuzzy}

A Figura 3 mostra os resultados do sistema Fuzzy construído a partir das informações das médias e desvios padrão das chuvas, definidos anteriormente, com o objetivo de identificar e prever o tipo de ZCAS atuante no Sul da Amazônia.

Neste sentido, ao fixar o valor $\mathrm{CN}$ fraca em $4,51 \mathrm{~mm} / \mathrm{dia}$ (Figura 3a) e variar a intensidade de CZ em 6,41 mm/dia (1), 12,5 $\mathrm{mm} / \mathrm{dia}$ (2) e 18,5 mm/dia (3) (coluna do meio), as classificações foram: ZCAS oceânica (a1), ZCAS oceânica (a2) e ZCAS costeira (a3). De modo semelhante na Figura 3b, fixa-se o valor de $\mathrm{CN}$ média $=9,4 \mathrm{~mm} / \mathrm{dia}$, variando as $\mathrm{CZ}$ fraca $=6,4 \mathrm{~mm} / \mathrm{dia}(1)$, 

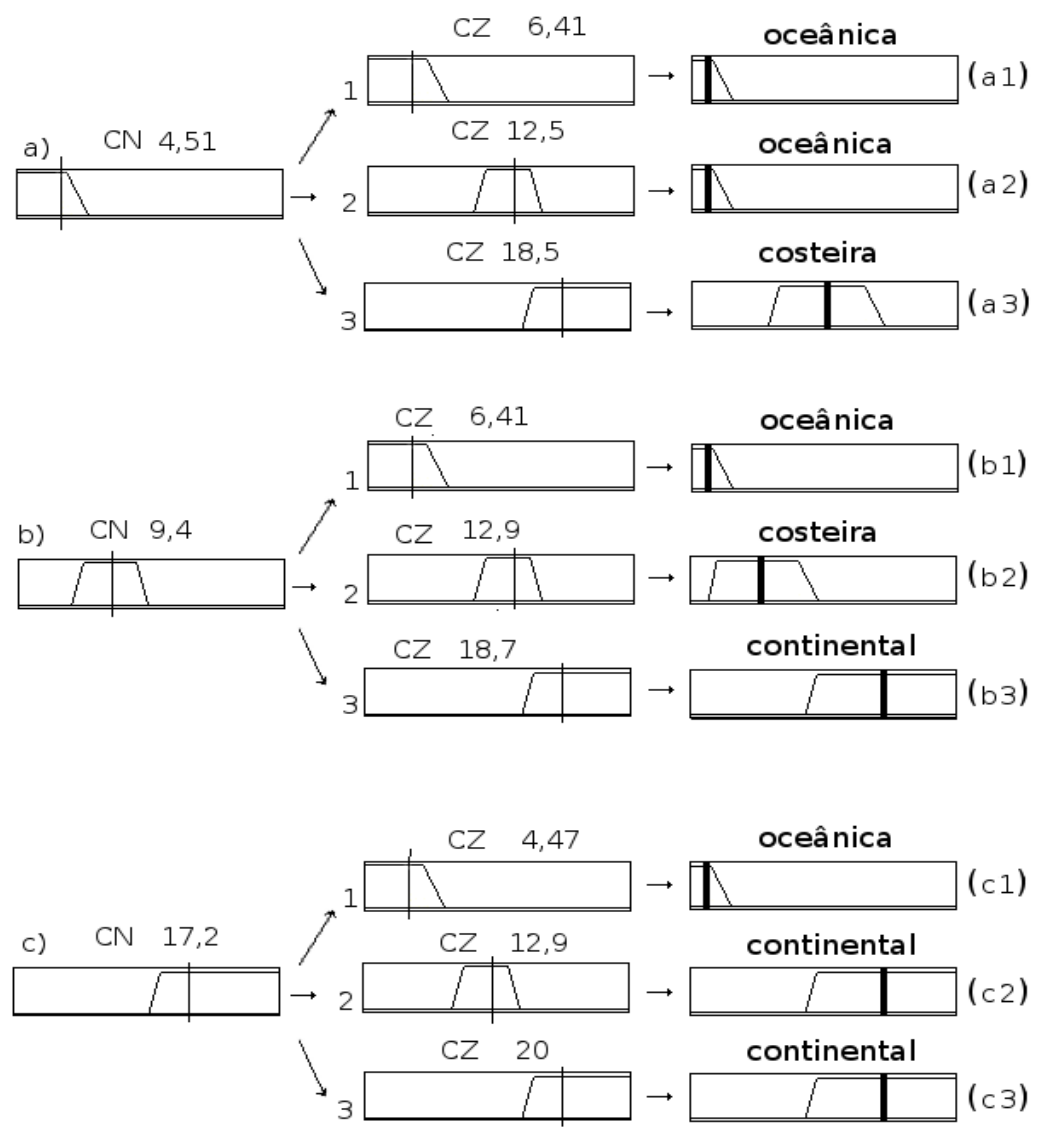

Figura 3 - Precipitação normal (CN) variando em fraca, média e forte (coluna da esquerda), chuvas de ZCAS (CZ) variando em fraca (1), forte(2) e intensa(3) (coluna do meio) e sua classificação em ai, bi e ci, i=1, 2 e 3, variando em oceânica, costeira e continental (coluna da direita). Unidades: $\mathrm{mm} / \mathrm{dia}$.

$\mathrm{CZ}$ forte $=12,9 \mathrm{~mm} / \mathrm{dia}(2)$ e $\mathrm{CZ}$ intensa $=18,7 \mathrm{~mm} / \mathrm{dia}(3)$, as classificações foram: ZCAS oceânica (b1), ZCAS costeira (b2) e ZCAS Amazônica (b3). Analogamente, na Figura 3c fixa-se o valor de $\mathrm{CN}$ forte $=17,2 \mathrm{~mm} /$ dia e são variadas as $\mathrm{CZ}$ em fraca $=4,47 \mathrm{~mm} / \mathrm{dia}(1), \mathrm{CZ}$ forte $=12,9 \mathrm{~mm} / \mathrm{dia}$ (2) e $\mathrm{CZ}$ intensa $=$ $20 \mathrm{~mm} /$ dia (3). As classificações foram: ZCAS oceânica (c1), ZCAS Amazônica (c2) e ZCAS Amazônica (c3). A resposta do modelo Fuzzy na classificação é mais consistente quando aumenta-se os valores da precipitação dos eventos de ZCAS em relação as chuvas normais, ou do mês em estudo. Essa variação na intensidade da precipitação sobre a área alvo, representada em três intensidades [fraca (1), forte (2) e intensa (3)], estão associados aos três tipos de ZCAS que influenciam o Sul da Amazônia.

\section{2 Classificação dos tipos de ZCAS}

O mesmo resultado pode ser observado quando utiliza-se a variável ROL na Figura 4. A região delimitada (linha tracejada) foi usada para destacar a posição climatológica dos episódio de ZCAS, semelhante aos trabalhos de Carvalho et al. (2004), Quadro (2011) e De Oliveira Vieira et al. (2012).
$\mathrm{Na}$ Figura 4a, destacam-se 8 (oito) eventos, correspondente a 28,6\% do total de ocorrências de ZCAS oceânica no Sul da Amazônia (ZCO). As anomalias significativas de ROL são observadas em três áreas distintas: a primeira no sul da Amazônia até o sul do continente, concordando com a presença dos JBN; a segunda, à noroeste do Brasil sofrendo interferência dos Andes; e a terceira a sudeste do Brasil no oceano Atlântico que é chamada de ZCO. A Figura 4b representa o padrão anômalo de ROL para 10 (dez) eventos, correspondente a 35,7\% do total de ocorrências de eventos de ZCAS costeiras (ZCC), mostrando três regiões com anomalias significativas: Uma sobre os Andes desde $15^{\circ} \mathrm{S}$ ao sul do continente; a outra na direção que é definida como ZCC; e na terceira, nota-se a presença da ZCIT no alto da figura. Nessa figura, a anomalia significativa se destaca sobre o nordeste e sudeste do país, a qual não foi observada anteriormente, principalmente no sul da Amazônia. Contudo, o padrão de ROL não cobre $1 / 2$ da área de estudo. Na Figura 4c é mostrado o padrão de anomalia significativo de ROL para os 10 (dez) eventos, correspondente a 35,7\% do total de ocorrências de eventos de ZCAS Amazônicas (ZCAM). Nessa figura, a região com maior diferença atua sobre todo o sul da 

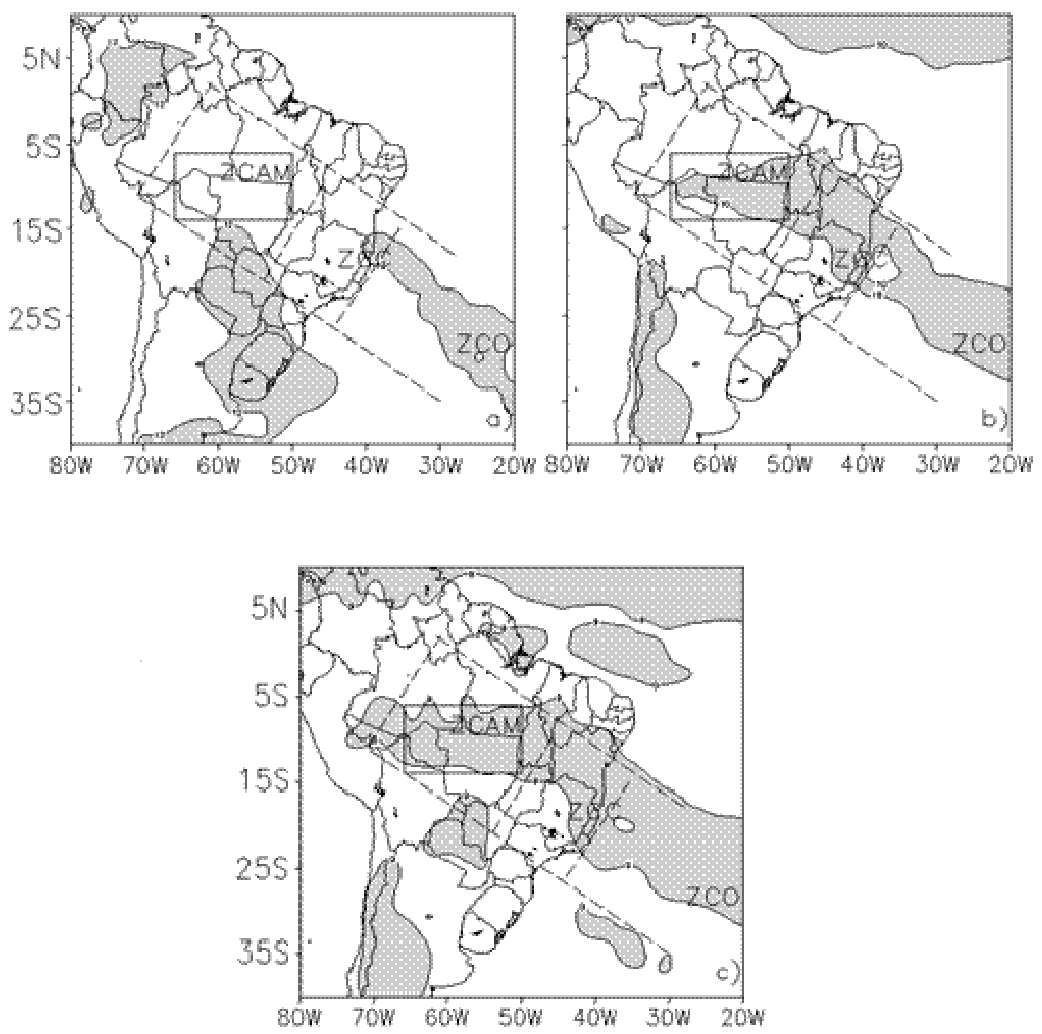

Figura 4 - Padrões de anomalias de ROL dos episódios de ZCAS classificados sobre a área-alvo (cor cinza), significância estatística em 95\% (linha de contorno). (a) ZCAS oceânica (ZCO), (b) ZCAS costeira (ZCC) e (c) ZCAS Amazônica (ZCAM).

Amazônia que é chamada de ZCAM, concentrando-se entre as latitudes $15^{\circ} \mathrm{S}$ a $5^{\circ} \mathrm{S}$. Em geral, esses padrões estão consistentes com os apresentados na Figura 4 e semelhantes ao encontrado por Carvalho et al. (2004) e De Oliveira Vieira et al. (2012).

Para ilustrar os padrões observados na Figura 4, são apresentados na Figura 5 compostos de temperatura de brilho para três eventos de ZCAS de acordo com as classificações dos eventos em ZCAM, ZCC e ZCO. O episódio considerado ZCAM na Figura 5a atuou no período de 28/02 a 04/03/2004 com duração de 5 dias, destacando uma região com menor temperatura de brilho sobre o Sul da Amazônia a nordeste/ sudeste brasileiro em direção ao Atlântico, semelhante ao da Figura 4c. Para o evento de 4 a 7/1/2007 (Figura 5b) com duração de 4 dias, a temperatura de brilho sobre as regiões do sul da Amazônia e a leste de MT não apresenta mesma intensidade em relação ao anterior. No entanto, os tipos de ZCAM e ZCC (Figura 5a e 5b) são os que contribuem com precipitações significativas acima de $47 \%$ e $23,5 \%$ em relação a média climatológica, respectivamente. Em relação a ZCO definida no período de 7 a 16/12/2006 e duração de 10 dias (Figura 5c), o padrão não apresenta a ZCAS bem caracterizada.. Contudo, observa-se uma homogeneidade na temperatura de brilho sobre toda a AS. Esse padrão favorece precipitação média não significativa no máximo $22 \%$ acima da climatologia sobre o Sul da Amazônia.

Foi demonstrado acima que o método Fuzzy têm potencial para classificar eventos ZCAS. Com intuito de explorar melhor essa questão, a aplicação do método para classificar o evento de ZCAS ocorrido entre 10 e 14/12/2010 é apresentado. A Figura 6 mostra o resultado da classificação Fuzzy do evento de ZCAS no período de 10 a 14/12/2010. Os resultados das precipitações médias sobre a área alvo do mês de dezembro e do episódio de ZCAS foram CN média $=8,6 \mathrm{~mm} /$ dia e CZ forte $=14,5 \mathrm{~mm} /$ dia, a função aplicada nesses valores $\mu$ (média, forte) classificou como ZCAS costeira. Nessa Figura 6a, destacam-se anomalia negativa de ROL igual a $-10 \mathrm{~W} / \mathrm{m}^{2}$ em grande parte da Amazônia, nordeste brasileiro e sobre o oceano Atlântico. Todavia o núcleo de maior intensidade $\left(\mathrm{ROL}=-30 \mathrm{~W} / \mathrm{m}^{2}\right)$ se destaca em três regiões do país: a primeira na região sul; a segunda no lado leste do Sul da Amazônia e a terceira cobrindo quase todo o Nordeste brasileiro, favorecendo atividade convectiva (Figura $6 \mathrm{~b}$ ) e posteriormente precipitação acima de $54 \%$ da média climatológica no Sul da Amazônia. Esse padrão de atividade convectiva (Figura 6b) favoreceu precipitação de 2 a 3 dias no nordeste brasileiro, sugerindo uma ligação da ZCAM e as chuvas no Nordeste na presença da ZCIT no verão austral. 

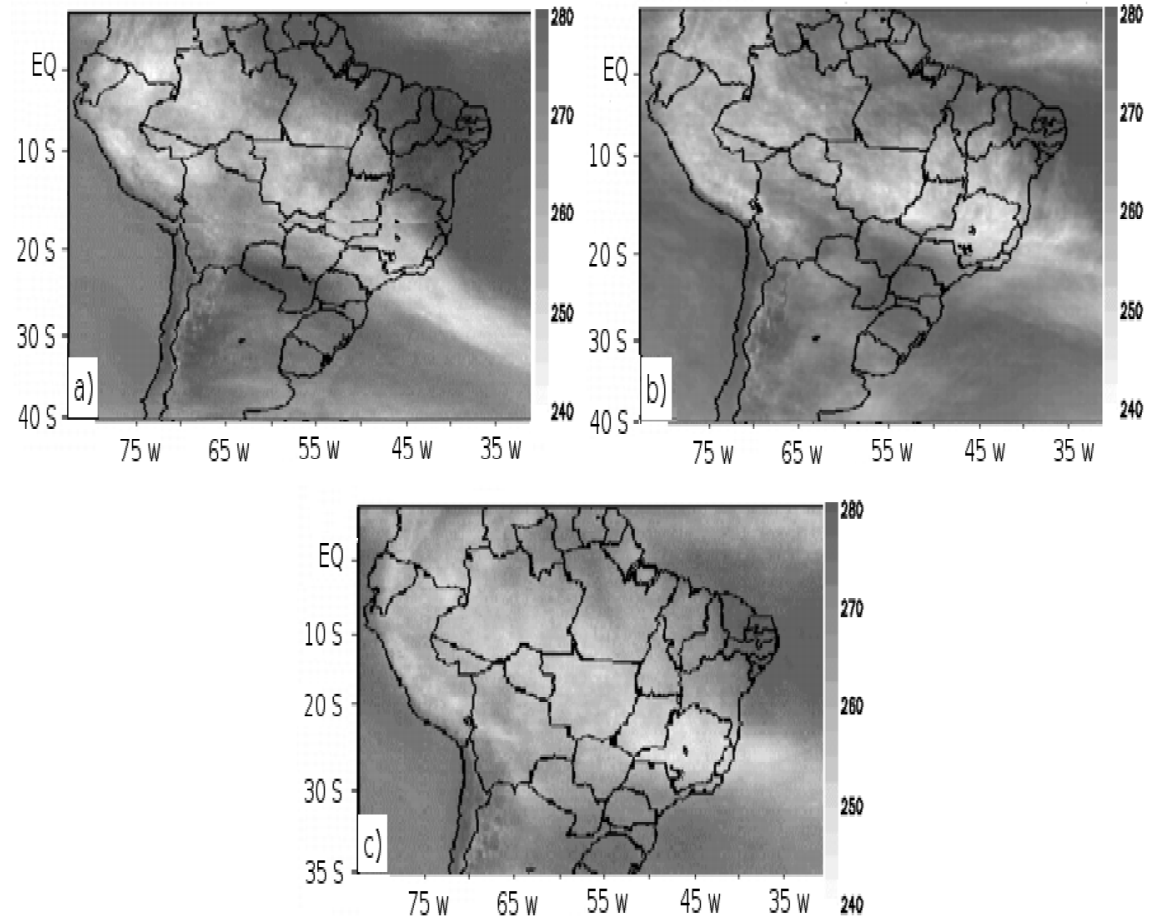

Figura 5: Compostos de imagens de Satélite GOES de Temperatura de brilho para três eventos, (a) ZCAM no período 29/01 a 09/02/2007, (b) ZCC no período 4 a 7/11/2007 e (c) ZCO no período 27/12/02 a 03/01/2003. Fonte: Boletim Climanálise - CPTEC.

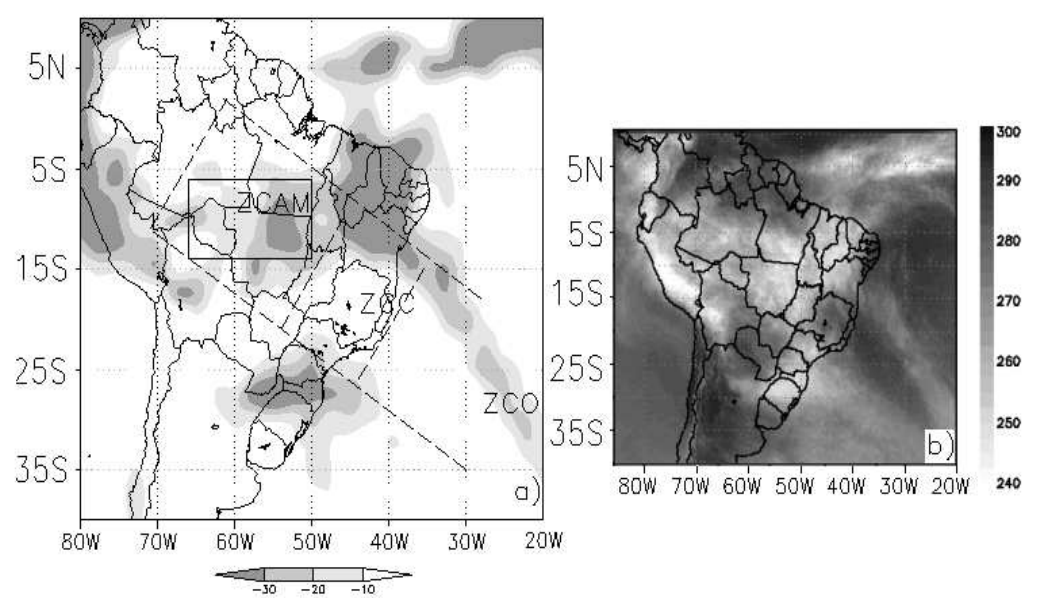

Figura 6 - (a) Anomalia de ROL sobre a média do mês e os dias de ZCAS. Período de 10 a 14/12/2010. Região retangular (cor preto) é a posição climatológica e área de estudo. ZCC em relação a série $\mathrm{S} 3$ e unidade em W/m². (b) Pêntadas de temperatura de brilho média (K) no período de 11 a 15/12/2010. (FONTE: Satélite GOES-12).

\section{3 ZCAS e os meses de atuação}

A Figura 7 apresenta os tipos de ZCAS (\%) classificados pelo método de inferência como também os meses (\%) de maior atuação durante os verões. Na Figura $7 \mathrm{a}$, o número total de ZCO atuantes nesta região correspondem a $8(28,6 \%)$ eventos, ZCC $10(35,7 \%)$ e ZCAM 10 (35,7\%), sendo que $71,4 \%$ dos episódios são ZCC e ZCAM. Esses contribuem com precipitação significativa cerca de $23,5 \%$ acima da média climatológica e em média 2 eventos por ano no Sul da Amazônia. Todavia, os episódios de ZCC e ZCAM são os responsáveis em causar transtornos sobre a população urbana e ribeirinha. Por outro lado, a análise da frequência dos eventos em relação aos meses de atuação na área alvo durante o período de estudo (Figura 7b), mostrou que a maior frequência de eventos ocorre nos meses de janeiro a março em média 7,3 eventos. Esses meses acumulam cerca de 78,6\% de todos os episódios. 
a)

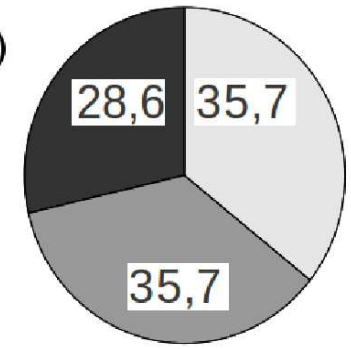

ZCAS $\square$ ZCAS $\square \quad$ ZCAS oceânica costeira Amazônica b)
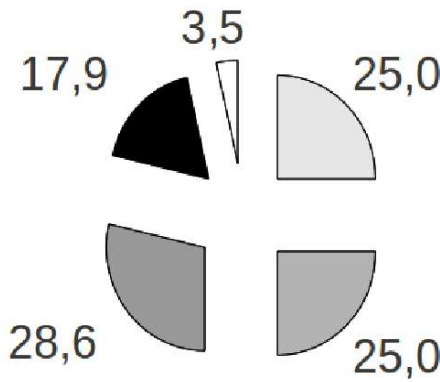

Novembro Dezembro $\square$ Janeiro $\square$ Fevereiro $\square$ Março

Figura 7 - Classificação dos episódios de ZCAS no sul da Amazônia. a) Tipos de ZCAS (\%) e b) meses de atuação (\%).

Tabela 1 - Frequência e percentagem da classificação dos eventos de ZCAS pelo método Fuzzy, referentes aos meses de atuação. ZCAS oceânica (ZCO), ZCAS costeira (ZCC) e ZCAS Amazônica (ZCAM).

\begin{tabular}{|l|c|c|c|c|c|}
\hline \multicolumn{1}{|c|}{ Mês } & ZCO $(\%)$ & ZCC $(\%)$ & ZCAM $(\%)$ & ZCO + ZCC $(\%)$ & ZCC + ZCAM(\%) \\
\hline Novembro & $1(12,5)$ & 0 & 0 & $1(5,6)$ & 0 \\
\hline Dezembro & $3(37,5)$ & $1(10)$ & $1(10)$ & $4(22,2)$ & $2(10)$ \\
\hline Janeiro & $2(25,0)$ & $2(20)$ & $4(40)$ & $4(22,2)$ & $6(30)$ \\
\hline Fevereiro & $1(12,5)$ & $3(30)$ & $4(40)$ & $4(22,2)$ & $7(35)$ \\
\hline Março & $1(12,5)$ & $4(40)$ & $1(10)$ & $5(27,8)$ & $5(25)$ \\
\hline Total & $8(100 \%)$ & $10(100 \%)$ & $10(100 \%)$ & $18(100 \%)$ & $20(100 \%)$ \\
\hline
\end{tabular}

Por outro lado, observa-se na Tabela 1 a classificação dos tipos de ZCAS que atuaram nos meses de NDJFM, apresentando a percentagem de ZCO mais concentrada nos meses de dezembro e janeiro, totalizando cerca de 5 eventos, as ZCC concentram-se nos meses de fevereiro e março, representando cerca de 7 eventos e as ZCAM concentram-se em janeiro e fevereiro, com 8 eventos. Essa tabela também apresentou dois casos interessantes no mês de novembro em relação aos eventos ZCC e ZCAM, os quais não atuaram nesse mês em todo o período de estudo no Sul da Amazônia. Todavia, nesse mês de novembro houve apenas um evento (ZCO) atuante em todo o período de estudo no Sul da Amazônia, resultado consistente com início do sistema de monção na AS.

O total de episódios de ZCC e ZCAM no sul da Amazônia é 20, concentrando-se basicamente nos meses de fevereiro com 7 eventos, janeiro com 6 eventos e março com 5 eventos, a diferença de apenas 1 evento entre esses meses, reflete diretamente no valor de precipitação significativa acima de $30 \%$ em relação a média climatológica.
A atuação das ZCO e ZCC sobre o Sul da Amazônia são bem marcantes, tendo em média $4(22,2 \%)$ eventos referentes aos meses de dezembro a março. Na Tabela 1, também se observa um aumento gradual de ZCAS nos meses de NDJFM, mas o mês de Abril não apresenta nenhum evento, demonstrando uma forte descontinuidade na atuação de ZCAS. Ao se analisar cada um dos boletins Climanálise referentes ao mês de abril durante o período de estudo, de fato, não foi observado um número significativo de episódios de ZCAS nesse mês. Portanto, as condições favoráveis para ocorrência de ZCAS no sul da Amazônia estão relacionadas com as ZCC e ZCAM, atuando principalmente nos meses de dezembro a março sumindo totalmente no mês de Abril.

\section{CONCLUSÃO}

Nesse estudo é apresentada uma aplicação do método Fuzzy para a classificação de ZCAS que atuam no sul da Amazônia. O modelo usado utiliza informações de precipitações 
climatológicas e àquelas referentes aos eventos de ZCAS para classificar esses eventos de acordo com sua intensidade e posição.

Os resultados mostram que, de modo geral, a Modelagem Fuzzy conseguiu classificar, a partir das intensidades das precipitações, os eventos de ZCAS em oceânica, costeira e Amazônica, bem como, determinar quais os meses de maior atuação desses eventos que influenciam as chuvas no Sul da Amazônia. A classificação dos 28 episódios de ZCAS selecionados no Sul da Amazônia durante o período de estudo, revelaram que $71,4 \%$ dos episódios são definidos como ZCAS costeiras (ZCC) e amazônicas (ZCAM). Esses dois tipos são os que mais contribuem com precipitação significativa, com valores maiores ou iguais a 30\% em relação à média climatológica. Assim, pode-se dizer que as ZCAS que satisfazem o Critério do Coeficiente de Eficiência da ZCAS em produzir precipitações fortes e intensas no Sul da Amazônia são as costeiras e Amazônicas. Ainda, a maior frequência de atuação desses eventos ocorreu nos meses de janeiro a março tendo em média 7,3 eventos mensais, totalizando em torno de $78,6 \%$ dos episódios.

Com base nesses resultados, pode-se dizer que o sistema de inferências proposto pode ser considerado como uma importante ferramenta de classificação de ZCAS para o sul da Amazônia, visto que o modelo utiliza somente informações de precipitação. O estudo mostrou que o método Fuzzy é uma ferramenta promissora na classificação qualitativa de sistemas sinóticos, como por exemplo, a ZCAS. Estudos anteriores, como o de Da Silva et al. 2007, também mostraram resultados promissores da utilização dessa técnica no auxílio à previsão sazonal de chuvas na região do Semiárido do Nordeste.

Para trabalhos futuros, sugere-se a busca de um entendimento sobre quais processos contribuem para o desenvolvimento desses tipos de ZCAS (ZCAM e ZCC) relacionadas com o Sul da Amazônia e quais as relações que as ZCAM podem ter com o VCN, AB e a ZCIT no nordeste brasileiro.

\section{AGRADECIMENTOS}

Os autores agradecem aos revisores pelos comentários e sugestões. O primeiro autor agradece a Universidade Federal do Amazonas (UFAM) pela liberação para cursar a Pós-Graduação do Instituto Nacional de Pesquisas da Amazônia em associação com a Universidade do Estado do Amazonas (INPA/UEA) e a CAPES pela bolsa de estudo concedida. O segundo, o terceiro e o quarto autores agradecem a Universidades Federal do Amazonas e a do Estado do Amazonas pelo suporte. O terceiro e quarto autores agradecem ao $\mathrm{CNPq}$ pelo apoio (Processo 305529/2012-9).

\section{REFERÊNCIAS}

BARROS, L. C. Sobre sistemas Dinâmicos Fuzzy-teoria e aplicações, Tese de Doutorado, IMECC-UNICAMP, Campinas. 1997.

CARVALHO, L .M. V., JONES, C., LEIBMANN, B. The South Atlantic Convergence Zone: Intensity, Form, Persistence, and Relationships with Intraseasonal to Interannual Activity and Extreme Rainfall. Journal of Climate, v. 17, p.88-108, 2004.

DE OLIVEIRA VIEIRA, S., SATYAMURTY, P., ANDREOLI, R. V. On the South Atlantic Convergence Zone affecting southern Amazonia in austral summer. Atmospheric Science Letters. DOI: 101002/asl.401. 2012.

FIGUEROA, S. N. SATYAMURTY, P, SILVA DIAS, P. L. Simulations of the summer circulation over the South American region with an Eta coordinate model. Journal Atmospheric Sciences. v. 52, p. 1573-1584. 1995.

HICKS, T.An Improved Fuzzy Logic System For Automated Short Term Aviation Weather Forecasts. In: AMS Conference on Aviation, Range, and Aerospace, 11., Hyannis, MA,p. 4-8. 2004. KODAMA, Y. Large-Scale Common Features of Subtropical Precipitation Zones (the Baiu Frontal Zone, the SPCZ, and the SACZ). Part I: Characteristics of Subtropical Frontal Zones. Journal of the Meteorological Society of Japan. v. 70, p. 813-836. 1992.

MAMDANI, E. H., ASSINLLAN, S. An Experimment in Linguistic Systhesis with a Fuzzy Logic Controller. International Journal of Man-Machine Studies. v.7, p.1-13. 1975.

QUADRO, M. F. L.; SILVA DIAS, M. A.; GONCALVES, L. G.; HERDIES, D. L.; BERBERY, E.H. Análise Climatológica da precipitação e do Transporte de umidade na região da ZCAS através da Nova geração de reanálises. Revista Brasileira de Meteorologia. v. 27, p.152-162, 2011.

RAO, V. B., HADA, K. Characteristics of rainfall over Brazil: Annual variations and connections with Southern Oscillation. Theoretical and Applied Climatology v. 42, p.81-91. 1990. 92-91467946/92-81241406

RAO, V. B., CAVALCANTI, I. F. A., HADA, K. Annual variation of rainfall over Brazil and water vapor characteristics over South America. Journal of Geophysical Research. v. 101, p. 26593-26551. 1996.

SHAO, J. Fuzzy Categorization of Weather Conditions for Thermal Mapping. Journal of Applied Meteorology. 39:1784-1790. 1999.

SATYAMURTY, P., NOBRE, C. A., SILVA DIAS, P. L. South America. In: Meteorology and Hydrology of the Southern Hemisphere. Eds. Karoly DJ, Vincent, D. G., American Meteorological Society, Meteorological Monograph. v. 49, p. 119-139. 1998. 
SATYAMURTY, P., DA COSTA, C. P. W., Manzi, A. O. Moisture source for the Amazon Basin: a study of contrasting years, Theoretical and Applied Climatology. DOI: 10.1007/s007040120637-7. Springer-Verlag. 2012.

SUGENO, M., KANG, G. T. Structure identification of Fuzzy model. Fuzzy Sets and Systems. v. 28, p.15-33. 1988.
VERA, C. et al. Towards a unified view of the American Monsoon systems. Journal of Climate. v.19, p.4977-5000. 2006.

ZADEH, L. A. Fuzzy Sets. Information and Control. v.8, p.338-353. 1965.

ZADEH, L. A. Fuzzy sets as basis for theory of possibility. Fuzzy Sets and systems. v.1, p.3-28. 1978. 